\title{
Non-equilibrium effects in chaperone-assisted translocation of a stiff polymer
}

\author{
Rouhollah Haji Abdolvahab \\ Physics Department, Iran University of Science and Technology (IUST), 16846-13114, \\ Tehran, Iran.
}

\begin{abstract}
Chaperone assisted biopolymer translocation is the main model proposed for translocation in vivo. A dynamical Monte Carlo method is used to simulate the translocation of a stiff homopolymer through a nanopore driven by chaperones. Chaperones are proteins that bind to the polymer near the wall and prevent its backsliding through Cis side. The important parameters include binding energy, size and the local concentration of the chaperones. The profile of these local concentrations, build up the chaperones distribution. Here we investigate the effects of binding energy, size and the exponential distribution of chaperones in their equilibration in each step of the polymer translocation needed for stable translocation time. The simulation results show that in case of chaperones with size of a monomer $(\lambda=1)$ and/or positive effective binding energy and/or uniform distribution, the chaperones binding equilibration rate/frequency is less than 5 times per monomer. However, in some special cases in exponential distribution of chaperones with size $\lambda>1$ and negative effective binding energy the equilibration rate will diverge to more than 20 times per monomer. We show that this non-equilibrium effect results in supper diffusion, seen before. Moreover, we confirm the equilibration process theoretically.
\end{abstract}

Keywords: Polymer translocation, First passage time, Chaperone distribution, Binding energy, Nanopore, supper-diffusion

Email address: rabdolvahab@gmail.com (Rouhollah Haji Abdolvahab) 


\section{Introduction}

Translocation of biomolecules through the nanopores Meller (2003) is one of the most important processes within biological cells. This is a ubiquitous process in cell metabolism. Protein's translocation through endoplasmic reticulum is an example. The polymer translocation also seen in proteins transport through organelles like mitochondria Alberts et al. (2002); Muthukumar (2007); Rapoport (2007). Translocation of messenger RNA through nuclear pore complexes in gene expression and in transcription through eukaryotic cells are two other biological instances Alberts et al. (2002). Translocation of DNA through protein channels covering the bacterial membrane amid phage infection is another example Bates et al. (2003); Dreiseikelmann (1994). Biotechnological examples also includes gene therapy, drug delivery and cheap rapid sequencing of the biopolymers Marzio and Kasianowicz (2003); Nakane et al. (2003); Branton et al. (2008); Cohen et al. (2012); Fanzio et al. (2012); Carson and Wanunu (2015); Liang and Zhang (2015). The experimental work of Kasianowicz et al. Kasianowicz et al. (1996) on ssRNA translocation through an $\alpha$-hemolysin channel was an influential work. Hereafter, there has been many experimental and theoretical works and simulations in polymer translocation Meller (2003); Panja et al. (2013); Sun and Luo (2014); Palyulin et al. (2014).

There are many different mechanisms to drive translocation of polymers. In vitro, people usually use a strong electric field to drive the translocation of highly charged biopolymers like single stranded DNA or RNA. Moreover, there could be many other parameters affecting the translocation such as crowding Gopinathan and Kim (2007); $\mathrm{Pu}$ et al. (2016), pressure and confinement Gravson and Molineux (2007); Panja and Molineux (2010); Molineux and Panja (2013); Palyulin et al. (2014). However, the most important model for the translocation in vivo is chaperone assisted translocation Tomkiewicz et al. (2007). This model with the name of Brownian ratchet mechanism was first proposed by Simon et al. in 1992 Simon et al. (1992). In this model proteins called chaperones are bound to the polymer in the Trans side and actively pull the polymer or just prevent its backsliding through the Cis side Alberts et al. (2002); Tomkiewicz et al. (2007). Later, the experiments of Matlack et al. in 1999 highlights the problem again Liebermeister et al. (2001); Elston (2002); Zandi et al. (2003). Subsequently, many theoretical and simulations struggled to have a better understanding of different aspects of the problem Palyulin et al. (2014); Ambjrnsson and Metzler 


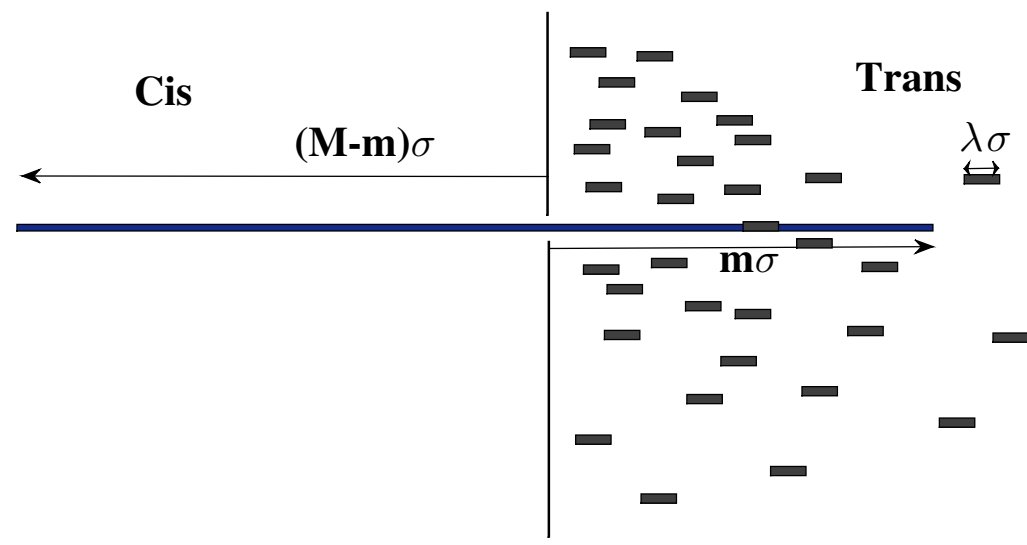

Figure 1: A stiff polymer is translocating from the Cis side (left) to the Trans side (right). Chaperones of size $\lambda \sigma$ are distributed in the Trans side. The total length of the polymer is $L=M \sigma . m$ monomers are no translocated to the right.

(2004); Abdolvahab et al. (2008, 2011b, a) ; Yu and Luo (2011, 2014); Wei-Ping Cao and Luo (2015); Suhonen and Linna (2016); Abdolvahab (2016).

There are many works on the non-equilibrium aspects of forced polymer translocation Sakaue (2007): Bhattacharya and Binder (2010); Saito and Sakaue (2013); Vollmer and de Haan (2016). This out of equilibrium property emerge as a result of force pulling the polymer through the Trans side. Here we investigate the chaperone assisted translocation which is used in vivo. Following our recent works we consider the chaperones exponential distribution effects on polymer translocation Abdolvahab (2016, 2017). We will show that even in the case of stiff polymer the distribution may induce the non-equilibrium effects in the translocation process.

In what follows we examine the chaperones binding rate effects on the translocation. Hereafter introducing our dynamical Monte Carlo simulation, we will discuss our simulation results in our main part of article in section 3 , Finally we will sum up our findings in the conclusion.

\section{Simulation}

\subsection{Theoretical model}

As the figure 1 shows, we simulate a stiff homopolymer consisted of $M$ monomer with size of $L=M \sigma$. Chaperones with the same size of $\lambda \sigma$ are distributed only in the Trans side. We suppose the $\lambda$ to be an integer Abdolvahab et al. (2011b); Ambjirnsson and Metzler (2004). They have 
local concentration which depends on their distribution. Moreover, there is a binding energy, positive or negative, between the chaperones and the polymer.

They bind (unbind) to (from) the polymer in the Trans side. The polymer always can go to the right. However, for backsliding of the polymer, its near the wall site must be unbound. The wall has no width Abdolvahab (2017). Binding of chaperones bias the translocation through the Trans side. The master equation for this process is written as:

$$
\begin{aligned}
\frac{\partial P(m, t)}{\partial t} & =\mathcal{W}^{+}(m-1) P(m-1, t-1) \\
& +\mathcal{W}^{-}(m+1) P(m+1, t+1) \\
& -\left(\mathcal{W}^{+}(m)+\mathcal{W}^{-}(m)\right) P(m, t)
\end{aligned}
$$

in which $\mathcal{W}^{ \pm}$are the transfer rates for translocating polymer to the right and left. $P(m, t)$ is the probability of finding polymer in time $t$ at condition in which $m$ monomer of it translocated to the right. Using transfer rates, $\mathcal{W}^{ \pm}$, and boundary conditions one could find the translocation time by calculating its mean first passage time Gardiner (2002).

\subsection{Describing the Monte-Carlo method}

We use a dynamical Monte Carlo method to simulate the translocation of a stiff polymer as follows. The polymer always can go to the right with probability of half. However, backsliding of the polymer is restricted. The polymer may come back through the Cis side only if there is not any chaperone bound to the polymer near the wall. Moreover, we use from the so called transmission boundary condition Redner (2001). It means reflective at first and absorbing at the end. As a result the polymer does not come back to the $\mathrm{Cis}$ when the first monomer is near the wall Abdolvahab (2017). Chaperones will try to bind/unbind in each step of the translocation by frequency $f$ per monomer per 40 . It means, for example, in the case of a polymer with $m=40$ and by the frequency $f=40$, the binding/unbinding process is one time. A monomer in the Trans side is selected randomly. If there is a chaperone bound to it we try to unbind it with its probability and vice versa (it will try to bind a chaperone accordingly). Due to our computational limits, we changed the frequency from 1 to $10^{3}$. 
Chaperones binding probability: There are three terms in the binding probability. Boltzmann distribution, which depend on the binding energy between chaperones and the polymer. Entropy linked to different patterns in which chaperones may distributed on the polymer and availability of the chaperones related to its local density Ambirnsson and Metzler (2004); Abdolvahab et al. (2011b). The second term is automatically comes in the simulation. In place of the binding energy, we define effective binding energy (EBE or $\mathscr{E}_{e f f}$ ) to combine the first and third term as Abdolvahab et al. (2011b) $\mathscr{E}_{\text {eff }} \equiv-\frac{1}{\lambda} \log \left[c_{0} v_{0} \exp \left(-\varepsilon / k_{B} T\right)\right]$, where $\varepsilon$ comes for the chaperone binding energy per monomer of the polymer. $c_{0}$ denotes the chaperone concentration, and $v_{0}$ stands for their volume Abdolvahab et al. (2011a); Abdolvahab (2017). Thus the binding and unbinding probabilities are written as:

$$
P_{\text {bind }}=\frac{\exp \left(-\sum_{i=1}^{\lambda} \mathscr{E}_{e f f}^{i}\right)}{1+\exp \left(-\sum_{i=1}^{\lambda} \mathscr{E}_{e f f}^{i}\right)}, P_{\text {unbind }}=\frac{1}{1+\exp \left(-\sum_{i=1}^{\lambda} \mathscr{E}_{e f f}^{i}\right)}
$$

The effective binding energy is changed from -4 to 4 and the polymer length is restricted to $M=50$. In order to reach to an acceptable error, we repeat the translocation process for at least $10^{4}$ times. Moreover, we use the chaperones of different sizes of $\lambda=1,2$ and 6 .

Chaperones distributions: For simplicity we restrict the chaperones to distributed only in the right part. We consider the exponential distribution with different rates, $\alpha$, for the chaperones and compare its results with the usual uniform distribution $(\alpha=0)$. In order to change the chaperones distribution in our Monte Carlo simulation it is enough to change the $\mathscr{E}_{e f f}$ to $\mathscr{E}_{e f f}+\alpha d$ in which $d$ is the distance (per monomer size) between the wall and the monomer in which we need its near chaperones concentration Abdolvahab (2016, 2017).

\section{Results and discussion}

\subsection{Mean translocation time}

We simulate 1 dimensional stiff homopolymer translocation through a nanopore using a dynamic Monte Carlo method in presence of the chaperones with different sizes and different spatial distributions. There are chaperones with distinct spatial distributions and various EBEs by monomers in the 

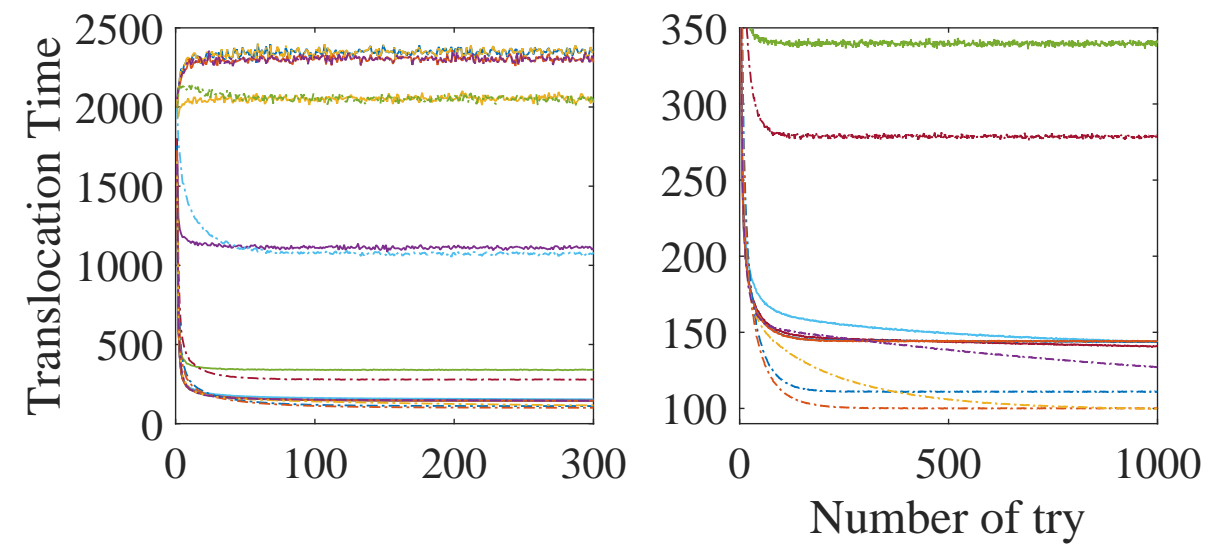

(a)

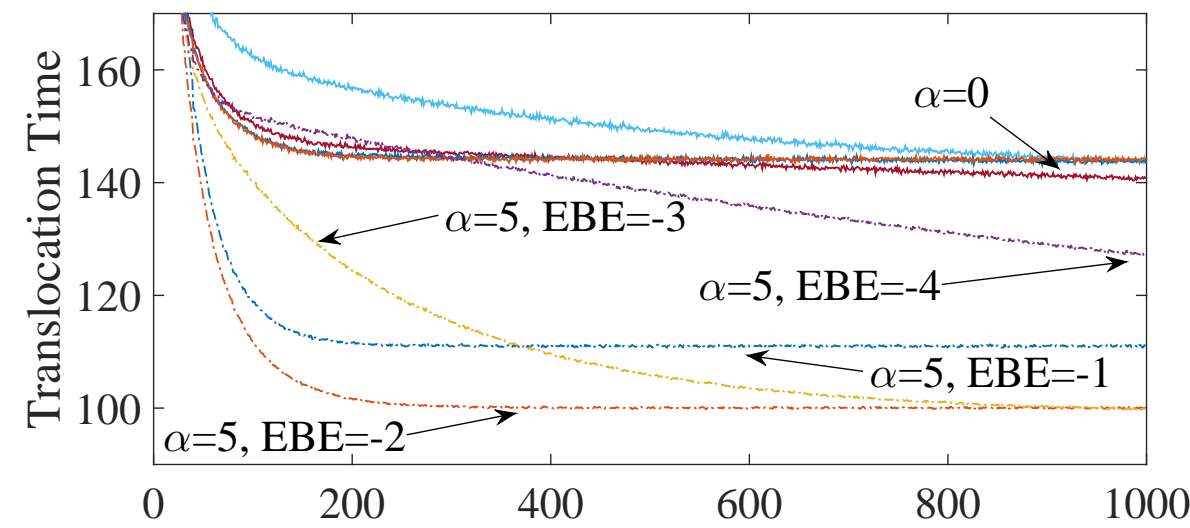

(b)

Figure 2: Translocation time of polymers, constructed of 50 monomers, versus try number. Chaperones size are $\lambda=2$ and exponential rates are $\alpha=0,5$. The dash-dotted lines are for $\alpha=5$ while the solid lines stand for $\alpha=0$. The right figure is a zoom on the left one. 
Trans side(There is not any chaperone in the Cis side.). Try number, or chaperones rate of binding (its frequency denotes by $f$ ), is an important parameter in calculating the translocation time of the polymer. In spite of this importance, there are few works on investigating its effects on the polymer translocation D'Orsogna et al. (2007). People suppose that due to interaction of the polymer with the pore and its size, the chaperones will reach to equilibrium in each step of the polymer translocation Ambjrnsson and Metzler (2004). In what follows we will show that although this is true for the case of uniform distribution of chaperones, in exponential distribution, the equilibration frequency will become large and the assumption is violated.

We translocate polymers in presence of chaperones of sizes $\lambda=1,2,6$ and spatial distributions of $\alpha=0,1,5,10$ and $E B E=-4:+4$. The figure 2 shows mean translocation time versus frequency or rate of the chaperones for different exponential chaperone distributions of $\alpha=0,5$ and different EBEs for chaperones of size $\lambda=2$. Different curves stand for different chaperones spatial distribution and/or different EBEs. The right figure is a zoom of the left one for faster polymers. Note that $\alpha=0$ means uniform spatial distribution of the chaperones.

The simulation results show that there are different regimes based on convexity and/or equilibrium rate:

- In large enough and positive EBEs the time versus rate curves are strictly ascending. They soon will reach to their equilibrium rates.

- In large enough and negative EBEs the time versus rate curves are strictly descending. They quickly will reach to their equilibrium rates usually but in some special cases the equilibrium rate will become quite large.

- In some intermediate energies, we will see a maximum in time versus rate curves.

In large positive EBEs, the chaperones do not prefer to bind to the polymer. Hence, the probability density function of finding the monomers bound are well asymmetric to the left (see the figure 31). In asymmetric density functions of this kind, mean is always in right and close to the most probable. We will show that in this situation increasing the fluctuation, will increase the $P_{\text {bind }}$ (near the wall binding probability). Consequently, by increasing the rate and decreasing the fluctuation, the translocation time will 


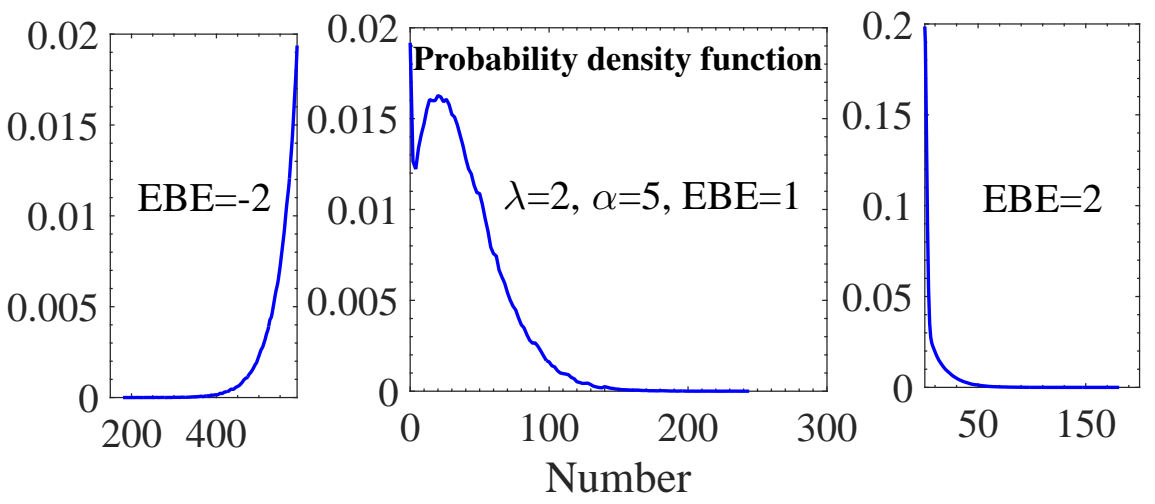

Figure 3: Probability distribution function of finding the near the wall monomer of the polymer bound is compared for chaperones of size $\lambda=2$ and exponential distribution with $\alpha=5$ for three different $\mathscr{E}_{\text {eff }}=-2,1,2$.

be increased (see the Appendix A.1). In contrast in large negative EBEs, increasing the frequency will cause the translocation time to be decreased (see the Appendix A.2).

\subsection{Equilibrium rate}

As discussed in the previous section, an important parameter in describing the polymer translocation is the equilibrium rate (the rate from which the translocation time does not change). In this regard, the polymers have two different behaviours. All the polymers with positive $\mathscr{E}_{e f f}$, which means the chaperones prefer not to bind to the polymer, have the small equilibrium rates. In contrast in the case of negative $\mathscr{E}_{e f f}$, the equilibrium rate could be relatively large. Indeed, our simulation results show that this non-equilibrium properties comes from the chaperones exponential distribution. We did not see any divergence in the uniform distribution of the chaperones (compare the plots in figure (4). The equilibrium rate of the polymers translocation in vicinity of the chaperones with different sizes of $\lambda=1,2,6$ and with chaperones spatial distribution with different exponential rates of $\alpha=0,1,5,10$ are plotted against $\mathscr{E}_{\text {eff }}$ in figure 4. As it shows there is not any important divergence in the cases of uniform distribution, $\alpha=0$, and/or chaperones with the size of a monomer, $\lambda=1$. To better understand this results one should find a more detailed description of the chaperones exponential distributions effects on the polymer translocation (see Abdolvahab (2017)). 


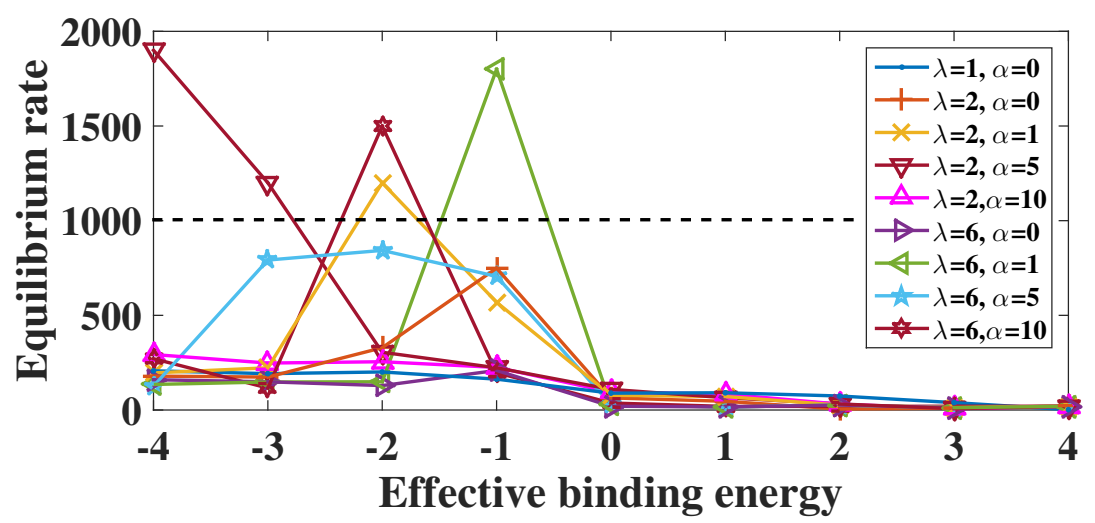

Figure 4: Equilibrium rates are plotted against EBEs for different binding sizes $\lambda=1,2,6$ and different chaperones distributions of $\alpha=0,1,5$ and10. The dashed line shows the maximum rate in the simulation. The points over the black dashed line are not real data and just estimated (based on figure 2).

It is pertinent to mention that our results paved the way for understanding the supper diffusion reported in our previous work Abdolvahab (2017). In that article we show that in some special case the scaling exponent of time vs polymer length, $\beta\left(T \sim M^{\beta}\right)$, becomes less than $1(\beta<1)$. It can be explained as follows. Increasing the polymer length will increase the time for chaperones to equilibrate. Consequently, the equilibration rate will be decreased. Hence, trying to calculate the scaling exponent $\beta$ before equilibrium will cause the translocation time increase less than the expected amount and the exponents could even become less than 1 .

\subsection{Mean waiting time}

Details of the translocation can be seen from its waiting times. Mean Waiting Time (MWT) of the translocation for chaperones of size $\lambda=2$ and $\mathscr{E}_{\text {eff }}=-4,4$ for uniform chaperone's distribution and 3 different rates of $f=10,300,900$ is shown in figure 5a, As it shows MWT for $\mathscr{E}_{\text {eff }}=4$ and for rates of $f \geq 300$ are decreasing linearly. However, for the same $\mathscr{E}_{\text {eff }}$ but for smaller rate of $f=10$, it starts from smaller amounts and it makes its translocation velocity faster. In contrast, in case of $\mathscr{E}_{\text {eff }}=-4$, the translocation velocity increase by increasing the rate from $f=10$ to $f=300,900$. As a result of the large binding probability the MWT for the rates $f=300,900$ becomes completely sawtooth shape.

As we saw in figure 2b, the translocation time for two different case of 


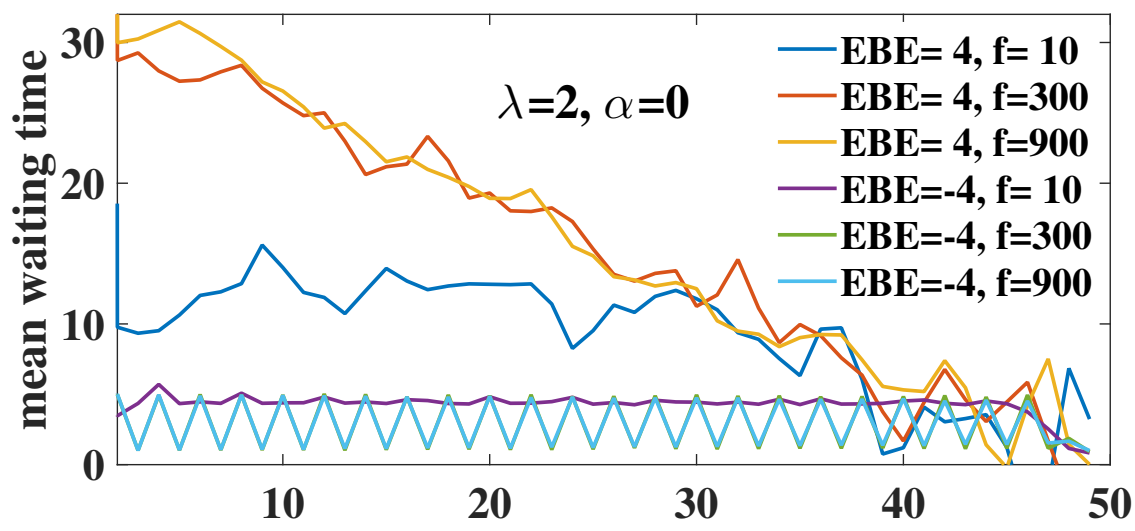

(a)

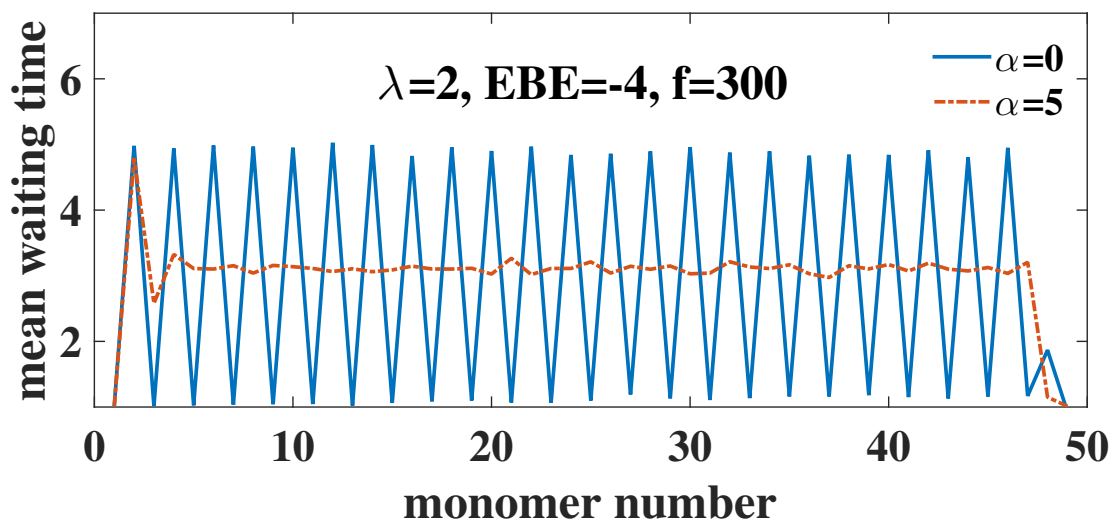

(b)

Figure 5: Mean waiting times of polymer translocation in different conditions are compared. In above figure, the chaperones have uniform distribution and different EBEs of $\mathscr{E}_{\text {eff }}=-4,4$ and rate frequencies of $f=10,300,900$ are compared(note that the chaperones with $\mathscr{E}_{\text {eff }}=-4$ and rate frequencies of $f=300,900$ are exactly the same.). In the figure $5 \mathrm{~b}$, we compare the uniform and exponential distribution of chaperones. 
uniform distribution and exponential distribution with $\alpha=5$, both with chaperones of size $\lambda=2$ and $\mathscr{E}_{\text {eff }}=-4$ in rates of $f=300$ equalize to each other. MWT for these two case is plotted in figure 5b. Because of the large negative $\mathscr{E}_{e f f}$, the chaperones bind to the polymer as right as it find a free place near the wall. In the case of uniform distribution availability of the chaperones are the same through the whole polymer which cause the sawtooth shape.However, in the case of exponential distribution, the chaperones may unbind from the polymer in further sites away from the wall. Consequently, the sawtooth shape becomes smooth or disappeared. On the other hand, in this case it takes time for the system to find its equilibrium and thus we see the translocation time is decreased by rate frequency and in $f=300$ it coincide with its uniform counterparts.

\section{Conclusions}

We simulate the translocation of stiff homopolymer through a nanopore driven by chaperones. We investigate specially the chaperones binding frequency and spatial distribution on the translocation time. Our results show that there are different patterns of equilibration in terms of chaperones size, effective binding energy and spatial distribution. In most cases the equilibrium is reaching soon (less than 10 try per monomer). However, an increase in equilibration frequency (more than 20 try per monomer) is seen in the cases of $\lambda>1, \mathscr{E}_{\text {eff }}<0$ and $\alpha>0$ roughly in the interval of $-6<\lambda \mathscr{E}_{\text {eff }}+\alpha<-1$. In larger amounts the chaperones do not prefer to bind and in less amounts the chaperones could not unbound from further sites. This result pave the way for understanding the supper diffusion reported in Abdolvahab (2017). We also strengthen our simulation results by theoretical discussion about the effect of chaperones binding rate on translocation time.

\section{Appendix A. Fluctuation in binding probability and translocation time}

Based on a master equation approach and using mean first passage time theory, we provide translocation time as follows Abdolvahab et al. (2011b):

$$
T=\frac{2 \tau_{0}}{P_{\text {bind }}}\left(N+1-\frac{1-P_{b i n d}}{P_{\text {bind }}}\left[1-\left(1-P_{b i n d}\right)^{N+1}\right]\right),
$$


where $\tau_{0}$ is the time takes for a bare polymer to translocate over distance of a monomer, $N$ is the total number of the monomers and $P_{\text {bind }}$ is the probability of the polymer to be bound near the wall. In the small and large amount of $P_{\text {bind }}$ one may approximate the equation A.1 as follows:

\section{Appendix A.1. Large binding probability}

In large enough binding probability $P_{\text {bind }} \simeq 1$ which occurs in large negative EBE, expansion of the equation A.1 leads to:

$$
T \simeq \frac{2 \tau_{0}}{P_{\text {bind }}}(N+1)
$$

Fluctuation of $P_{b i n d}$ which shows itself in translocation time can be investigated as follows. Admit the change in $P_{\text {bind }}$ to be of order $\delta ; P_{\text {bind }} \rightarrow$ $P_{b i n d} \pm \delta$. We then average over this range by integration:

$$
\bar{T}_{\text {large }} \simeq \frac{1}{2 \delta}\left(\int_{P_{\text {bind }}-\delta}^{P_{\text {bind }}+\delta} \frac{2 \tau_{0}}{P}(N+1) d P\right) \simeq \frac{2 \tau_{0}(N+1)}{P_{\text {bind }}-\delta},
$$

where $\bar{T}_{\text {large }}$ is average of $T$ over fluctuations of $P_{\text {bind }}$ for large binding probabilities. As expected from our simulation results, it shows that increasing the chaperones binding rate which decreases the fluctuation $\delta$, will reduce the mean translocation time.

\section{Appendix A.2. Small binding probability}

In contrast to the previous section, in small binding probability, expansion of equation A.1 results in:

$$
\begin{aligned}
& T\left(P_{b i n d}\right)=2 \tau_{0}(N+1) \times\left(C_{0}(N)-C_{1}(N) P_{b i n d}+C_{2}(N) P_{b i n d}^{2}-\cdots\right), \\
& C_{0}(N)=1+\frac{N}{2}, C_{1}(N)=\frac{N}{2}\left(1+\frac{N-1}{3}\right), C_{2}(N)=\frac{N(N-1)}{3 !}\left(1+\frac{N-2}{4}\right) .
\end{aligned}
$$

Note that this approximation is true when $N P_{\text {bind }} \ll 1$ and the important parameter here is the Péclet number not $P_{\text {bind }}$ itself (see Abdolvahab et al. (2011a) for more detail). As the figure 3 shows, the binding probability distribution is exponential. Let assume its exponential determines by parameter $a ; P\left(P_{b i n d}\right) \propto \exp \left(-a P_{b i n d}\right)$. Averaging of the translocation time over fluctuations of $P_{\text {bind }}$ leads to: 


$$
\begin{aligned}
& Z \equiv\left(\int_{P_{\text {bind }}-\delta}^{P_{\text {bind }}+\delta} \exp (-a P) d P\right) \\
& \Rightarrow \bar{P}_{\text {bind }}=-\frac{\partial}{\partial a} \ln (Z) \simeq-\frac{\partial}{\partial a} \ln \left(2 \delta \exp \left(-a P_{\text {bind }}\right)\left(1-(a \delta)^{2}\right)\right) \simeq P_{\text {bind }}+2 a \delta^{2} \\
& \bar{T}_{\text {small }}-T=\frac{1}{2 \delta}\left(\int_{P_{\text {bind }}-\delta}^{P_{\text {bind }}+\delta} T(P) \exp (-a P) d P\right)-T\left(P_{\text {bind }}\right) \\
& \Rightarrow \bar{T}_{\text {small }}-T \simeq-C_{1}(N) 2 a \delta^{2} .
\end{aligned}
$$

Increasing the chaperones binding rate will decrease the fluctuation over $P_{\text {bind }}, \delta$, and as a result the mean translocation time, $\bar{T}_{\text {small }}$, will be increased. It is in place to note here that the $E B E$ in which the regimes changed, say $E B E_{0}$, may be obtained by comparison of second and third term in equation A.4:

$$
C_{1}(N) P_{\text {bind }}>C_{2}(N) P_{b i n d}^{2} \Rightarrow \frac{N}{3} P_{b i n d}<1 .
$$

Presume the binding probability to be proportional to its Boltzmann distribution $\left(P_{\text {bind }} \propto \exp (-\lambda E B E)\right)$ leads us to $E B E_{0} \simeq \frac{1}{\lambda} \ln \left(\frac{N}{3}\right)$. As an example in case of $\lambda=2$ and $N=50, E B E_{0} \simeq 1.4$ which is compatible with the simulation results (see e.g. figure 2a).

\section{References}

\section{References}

Abdolvahab, R. H., 2016. Investigating binding particles distribution effects on polymer translocation through nanopore. Physics Letters A 380, 10231030 .

Abdolvahab, R. H., 2017. Chaperone driven polymer translocation through nanopore: spatial distribution and binding energy. The European Physical Journal E 40, 41.

Abdolvahab, R. H., Ejtehadi, M. R., Metzler, R., 2011a. First passage time distribution of chaperone driven polymer translocation through a nanopore: Homopolymer and heteropolymer cases. Journal Of Chemical Physics 135, 5102. 
Abdolvahab, R. H., Ejtehadi, M. R., Metzler, R., 2011b. Sequence dependence of the binding energy in chaperone-driven polymer translocation through a nanopore. Physical Review E 83, 011902.

Abdolvahab, R. H., Roshani, F., Nourmohammad, A., Sahimi, M., Tabar, M. R. R., 2008. Analytical and numerical studies of sequence dependence of passage times for translocation of heterobiopolymers through nanopores. Journal of Chemical Physics 129 (235102), 1-8.

Alberts, B., Johnson, A., Lewis, J., Raff, M., Roberts, K., Walter, P., 2002. Molecular Biology of the Cell. Garland Publishing, New York.

Ambjrnsson, T., Metzler, R., 2004. Chaperone-assisted translocation. Physical Biology 1, 77.

Bates, M., Burns, M., Meller, A., 2003. Dynamics of dna molecules in a membrane channel probed by active control techniques. Biophysical journal 84 (4), 2366-2372.

Bhattacharya, A., Binder, K., 2010. Out-of-equilibrium characteristics of a forced translocating chain through a nanopore. Physical Review E 81 (4), 041804 .

Branton, D., Branton, D., Deamer, D. W., Marziali, A., Bayley, H., Benner, S. A., Butler, T., Ventra, M. D., Garaj, S., Hibbs, A., Huang, X., Jovanovich, S. B., Krstic, P. S., Lindsay, S., Ling, X. S., Mastrangelo, C. H., Meller, A., Oliver, J. S., Pershin, Y. V., Ramsey, J. M., Riehn, R., Soni, G. V., Tabard-Cossa, V., Wanunu, M., Wiggin, M., Schloss, J. A., Deamer, D. W., Marziali, A., Bayley, H., Benner, S. A., Butler, T., Ventra, M. D., Garaj, S., Hibbs, A., Huang, X., Jovanovich, S. B., Krsticand, P. S., Lindsay, S., Ling, X. S., Mastrangelo, C. H., Meller, A., Oliver, J. S., Pershin, Y. V., Ramsey, J. M., Riehn, R., Soni, G. V., Tabard-Cossa, V., Wanunu, M., Wiggin, M., Schloss, J. A., 2008. The potential and challenges of nanopore sequencing. Nature Biotechnology 20, 1146.

Carson, S., Wanunu, M., 2015. Modulating dna translocation by a controlled deformation of a pdms nanochannel device. Nanotechnology 26, 074004.

Cohen, J. A., Chaudhuri, A., Golestanian, R., 2012. Stochastic sensing of polynucleotides using patterned nanopores. Physical Review X 2, 2160. 
D’Orsogna, M. R., Chou, T., Antal, T., 2007. Exact steady-state velocity of ratchets driven by random sequential adsorption. Journal of Physics A: Mathematical and Theoretical 40 (21), 5575.

Dreiseikelmann, B., 1994. Translocation of dna across bacterial membranes. Microbiological reviews 58 (3), 293-316.

Elston, T. C., 2002. The brownian ratchet and power stroke models for posttranslational protein translocation into the endoplasmic reticulum. Biophysical Journal 82, 1239.

Fanzio, P., Manneschi, C., Angeli, E., Mussi, V., Firpo, G., Ceseracciu, L., Repetto, L., Valbusa, U., 2012. Modulating dna translocation by a controlled deformation of a pdms nanochannel device. Scientific Report 2, 791.

Gardiner, C. W., 2002. Handbook of Stochastic Methods for Physics, Chemistry and the Natural Sciences, 2nd Edition. Vol. 13 of Synergetics. Springer, New York.

Gopinathan, A., Kim, Y. W., 2007. Polymer translocation in crowded environments. Physical Review Letters 99, 228106.

Grayson, P., Molineux, I. J., 2007. Is phage dna injectedinto cellsbiologists and physicists can agree. Current opinion in microbiology 10 (4), 401-409.

Kasianowicz, J. J., Brandin, E., Branton, D., Deamer, D. W., 1996. Characterization of individual polynucleotide molecules using a membrane channel. Proceedings of the National Academy of Sciences U.S.A. 93, 13770.

Liang, F., Zhang, P., 2015. Nanopore dna sequencing: Are we there yet? Science Bulletin 60, 296.

Liebermeister, W., Rapoport, T. A., Heinrich, R., 2001. Ratcheting in posttranslational protein translocation: a mathematical model. Journal of Molecular Biology 305, 643.

Marzio, E. D., Kasianowicz, J. J., 2003. Phase transitions within the isolated polymer molecule: Coupling of the polymer threading a membrane transition to the helix-random coil, the collapse, the adsorption, and the equilibrium polymerization transitions. Journal of Chemical Physics 119, 6378 . 
Meller, A., 4 2003. Dynamics of polynucleotide transport through nanometrescale pores. Journal of Physics: Condensed Matter 15, R581.

Molineux, I. J., Panja, D., 2013. Popping the cork: mechanisms of phage genome ejection. Nature reviews. Microbiology 11 (3), 194.

Muthukumar, M., 2007. Mechanism of dna transport through pores. Annual Review of Biophysics and Biomolecular Structure 36, 435.

Nakane, J. J., Akeson, M., Marziali, A., 2003. Nanopore sensors for nucleic acid analysis. Journal of Physics: Condensed Matter 15 (32), R1365.

Palyulin, V. V., Ala-Nissila, T., Metzler, R., 2014. Modulating dna translocation by a controlled deformation of a pdms nanochannel device. Soft Matter 10, 9016.

Panja, D., Barkema, G. T., Kolomeisky, A. B., 2013. Through the eye of the needle: recent advances in understanding biopolymer translocation. Journal of Physics: Condensed Matter 25 (41), 413101.

Panja, D., Molineux, I. J., 2010. Dynamics of bacteriophage genome ejection in vitro and in vivo. Physical biology 7 (4), 045006.

Pu, M., Jiang, H., Hou, Z., 2016. Polymer translocation through nanopore into active bath. The Journal of chemical physics 145 (17), 174902.

Rapoport, T. A., 2007. Protein translocation across the eukaryotic endoplasmic reticulum and bacterial plasma membranes. Nature 450, 663.

Redner, S., 2001. A guide to first-passage processes. Cambridge University Press, Cambridge UK.

Saito, T., Sakaue, T., 2013. Cis-trans dynamical asymmetry in driven polymer translocation. Physical Review E 88 (4), 042606.

Sakaue, T., 2007. Nonequilibrium dynamics of polymer translocation and straightening. Physical Review E 76 (2), 021803.

Simon, S. F., Peskin, C. S., Oster, G. F., 5 1992. What drives the translocation of proteins? Proc. Natl Acad. Sci.USA 89, 3770. 
Suhonen, P. M., Linna, R. P., 2016. Chaperone-assisted translocation of flexible polymers in three dimensions. Physical Review E 93, 012406.

Sun, L.-Z., Luo, M.-B., 2014. Langevin dynamics simulation on the translocation of polymer through $\alpha$-hemolysin pore. Journal of Physics: Condensed Matter 26 (41), 415101.

Tomkiewicz, D., Nouwen, N., Driessen, A. J. M., 2007. Pushing, pulling and trapping modes of motor protein supported protein translocation. Federation of European Biochemical Societies Letters 581 (15), 2820-2828.

Vollmer, S. C., de Haan, H. W., 2016. Translocation is a nonequilibrium process at all stages: Simulating the capture and translocation of a polymer by a nanopore. The Journal of chemical physics 145 (15), 154902.

Wei-Ping Cao, Q.-B. R., Luo, M.-B., 2015. Translocation of polymers into crowded media with dynamic attractive nanoparticles. Physical Review E 92, 012603.

Yu, W., Luo, K., 2011. Chaperone-assisted translocation of a polymer through a nanopore. Journal of the American Chemical Society 133, 13565.

Yu, W., Luo, K., 2014. Polymer translocation through a nanopore driven by binding particles: Influence of chain rigidity. Physical Review E 90, 042708 .

Zandi, R., Reguera, D., Rudnick, J., Gelbart, W. M., 7 2003. What drives the translocation of stiff chains? Proceedings of the National Academy of Sciences USA 100, 8649. 